\title{
What is new in dravet syndrome?
}

\author{
Lucia M. Sur ${ }^{1,2}$, Remus Gaga ${ }^{3}$, Gabriel Samasca ${ }^{1,3}$, Cornel Aldea ${ }^{3}$, Genel Sur ${ }^{3}$, Iulia Lupan ${ }^{3,4}$ \\ 1"Iuliu Hatieganu" University of Medicine and Pharmacy, Cluj-Napoca, Romania \\ ${ }^{2}$ Pediatrics Department, Emergency Clinical Hospital for Children, Cluj-Napoca Romania. \\ ${ }^{3}$ Molecular Biology Department, "Babes Bolyai" University, Cluj-Napoca, Romania \\ ${ }^{4}$ Interdisciplinary Research Institute on Bio-Nano-Science, "Babes Bolyai" University, Cluj-Napoca, Romania
}

\begin{abstract}
The pathology of Dravet syndrome is of particular importance in children. In this article we analyze the involvement
of the SCN1A gene in Dravet syndrome, we approach the new treatment strategies in Dravet syndrome.
Keywords: myoclonic epilepsy, Dravet syndrome, febrile seizures, children, rare genetic
\end{abstract} disease

\section{INTRODUCTION}

Dravet syndrome (DS), also known as severe myoclonic epilepsy of infants, was first described by Charlotte Dravet in 1978. It's a rare genetic disease affecting between 1 in 15,000 and 1 in 40,000 children. DS is characterized by seizures that start to manifest before 1 year of age, usually during fever. Typically, the seizures involve half the body, lateralization can vary between episodes and it often evolves into status epilepticus. New types of seizures may appear between 1 and 2 years of age: absence seizures, myoclonic seizures, or generalized tonic-clonic seizures. Pure tonic seizures are rare. Rises in body temperature continue to cause seizures throughout childhood, these seizures being difficult to control [1].

Psychomotor development is usually normal until 1 year of age, later, alongside the diversification of the seizure types; mental stagnation will usually be present. Patients frequently develop severe mental retardation; however, the extent of the retardation greatly depends on seizure control.
Most cases associate attention deficit hyperactivity disorder, autistic traits, and motor involvement such as ataxia, tremor, dysarthria, and bilateral pyramidal signs [2-4]. Normal psychomotor development is also possible [5].

Electroencephalogram (EEG) findings are usually normal during the first year, later generalized and multifocal abnormalities can be seen. No EEG pattern alone is diagnostic for DS [6]. Brain imaging is normal until 1 year of age and later only $10 \%$ of patients may present cerebral atrophy or hippocampal sclerosis. The differential diagnosis of DS includes febrile seizures, Lennox-Gastaut syndrome, and myoclonic-astatic epilepsy [7].

We aimed to analyze SCN1A gene implication in DS. Also, we wanted to approach new treatment strategies in DS. We searched in the literature and we analyzed only the relevant articles.

\section{ANALYSIS OF THE SCN1A GENE}

Approximately $80 \%$ of DS patients have a mutation of the SCN1A gene, which is involved in the 
function of sodium channels. Over 700 different mutations of the SCN1A gene were found, which might explain the large variation within the phenotype. Currently, no clear correlation has been found between the exact genotype and the phenotype. Most patients have a de novo mutation affecting the SCN1A gene. Only $5 \%$ of patients inherit the mutation, however, $30-50 \%$ of patients have a family history of febrile seizures. Because of the varied phenotype associated with the SCN1A gene mutation, the term DS is preferred, rather than severe myoclonic epilepsy of infants.

Mutations in the SCN1A gene are associated with several epileptic syndromes. These channelopathies are associated with a broad epileptic spectrum, from genetic epilepsy with febrile seizures (GEFS) to developmental encephalopathy (DEE). Hemiplegic migraine and autism spectrum disorders may also occur within these SNC1A changes. DS is part of epileptiform developmental epilepsy (ED), which begins early, usually through a febrile episode. Convulsions can take on different aspects but the most characteristic is the myoclonic ones, they can also be localized clonic, generalized tonic-clonic. EEG and imaging investigations are normal at onset in DS. Psychomotor development is normal in the first year of life. Epilepsy is resistant to drugs, even in polytherapy.

After the age of 1 year, intellectual regression usually occurs. Most mutations in the SNC1A gene occur de novo. The differential diagnosis is made with the entities derived from the SCN-1A mutations of the sodium channel. We mention here GEFS and DEE. Within this DEE there is a spectrum of severity ranging from atonic myoclonic epilepsy (MAE), epilepsy of children with focal migratory seizures (EIMFS), hemiplegic migraine epilepsy, and autism spectrum changes.

MAE is a form of generalized genetic epilepsy that begins between 1 and 7 months. The seizures have a variable appearance and the symptoms include "fall attacks".

EIMFS begins at 2 months and is characterized by focal seizures that migrate from one hemisphere to another. Seizures can be very common and affect a child's development. Mutations in the SCN1A gene are rare, with the major causative gene for this syndrome being KCNT1 in almost half of the cases.
SCN1A DEE mutation form with early-onset starts at 8-12 weeks and is the most severe form of epilepsy related to the SCN1A genes. Seizures can be localized, generalized tonic-clonic, spasms. Also, these patients show a delay in psychomotor development.

Genetic epilepsy with febrile ascents occurs in families with more children with a genetic inheritance of seizures, there is a low seizure threshold and SNC1A mutations have been found in $20 \%$ of those diagnosed.

Common epilepsies are forms of epilepsy. It has been tried to see if SCN1A mutations are involved in the occurrence of and it has been found that there are possibilities of involvement of these mutations [8-9].

\section{NEW TREATMENT STRATEGIES}

The main purpose of the treatment in DS is seizure control, mainly to reduce the number of seizures. Medication that blocks sodium channels such as carbamazepine and phenytoin may exacerbate seizures and is, therefore, to be avoided. An exacerbation of seizures under these types of medication should raise the suspicion of DS. Medication that was proven useful in DS consists of topiramate, valproic acid, benzodiazepines, and levetiracetam. Non-pharmacologic treatment options such as ketogenic diet and vagus nerve stimulation might also be effective in some cases. In 2018 the FDA has approved the use of cannabidiol in DS for reducing seizure frequency.

Avoiding seizure triggers is vital for these patients. The most frequent triggers consist of changes in the body and environmental temperature, acute diseases, and stress. Rises in body temperatures should be treated aggressively whenever they appear. Antipyretics should be used aggressively whenever fever is present [10].

Among the new treatments that can bring beneficial results, we mention fenfluramine, this treatment may reduce monthly convulsive seizure frequency in patients with DS who have poor seizure control with other antiepileptic drug regimens. However, the side effects and possible associated cardiovascular and ophthalmic diseases must be taken into account [11-15].

Considering that often seizures in DS are drug-resistant, treatment can prove complicated. 
Patients often require permanent supervision, the disease severely affecting both the patient's and the family's life quality. Devices that proved useful to the families of DS consist of video monitoring, cooling vests, alarms that go off when seizures take place, pulse oximetry, and even helmets. Family psychotherapy is recommended whenever possible. The average lifespan of DS patients is 8 years, the most common cause of death being sudden unexpected death in epilepsy, followed by status epilepticus $[16,17]$.

\section{CONCLUSIONS}

DS has different phenotypes and manifestations probably due to different mutations (over $700 \mathrm{SN}$ C1A mutations). DS is not pure epilepsy but also associates intellectual, mental, and motor developmental retardation. DS has a negative impact on both children and family members.

\section{Acknowledgement}

All authors contributed equally to this work.

Conflict of interest: none declared Financial support: none declared

\section{REFERENCES}

1. Hirtz D, Thurman DJ, Gwinn-Hardy K, Mohamed M, Chaudhuri AR, Zalutsky R. How common are the "common" neurologic disorders? Neurology. 2007 Jan 30;68(5):326-37.

2. Wu YW, Sullivan J, McDaniel SS, et al. Incidence of DS in a US Population. Pediatrics. 2015;136:e1310.

3. Chieffo D, Battaglia D, Lettori D, Del Re M. Neuropsychological development in children with DS. Epilepsy Res. 2011;95(1-2):86-93.

4. Jansen FE, Sadleir LG, Harkin LA, et al. Severe myoclonic epilepsy of infancy (DS): recognition and diagnosis in adults. Neurology. 2006;67:2224.

5. Sur L, Samasca G, Sur G, Gaga R, Aldea C. A Mutation in the SCN1A Gene With a Peculiar Course: A Case Report. Cureus. 2021;13(2):e13612.

6. Specchio N, Balestri M, Trivisano M, et al. Electroencephalographic features in DS: five-year follow-up study in 22 patients. $J$ Child Neurol. 2012;27:439.

7. Proposal for revised classification of epilepsies and epileptic syndromes. Commission on Classification and Terminology of the International League Against Epilepsy. Epilepsia. 1989 Jul-Aug; 30(4):389-99.

8. Claes L, Del-Favero J, Ceulemans B, et al. De novo mutations in the sodium-channel gene SCN1A cause severe myoclonic epilepsy of infancy. Am J Hum Genet. 2001;68:1327.

9. Yeşil G, Aralaşmak A, Akyüz E, et al. Expanding the Phenotype of Homozygous KCNMA1 Mutations; Dyskinesia, Epilepsy, Intellectual
Disability, Cerebellar and Corticospinal Tract Atrophy. Balkan Med J. 2018;35:336-339.

10. Wirrell EC, Laux L, Donner E, et al. Optimizing the Diagnosis and Management of DS:Recommendations From a North American Consensus Panel. Pediatr Neurol. 2017;68:18.

11. Wirrell EC, Nabbout R. Recent Advances in the Drug Treatment of Dravet Syndrome. CNS Drugs. 2019;33:867-881.

12. Nabbout R, Mistry A, Zuberi S, et al. Fenfluramine for TreatmentResistant Seizures in Patients With Dravet Syndrome Receiving Stiripentol-Inclusive Regimens: A Randomized Clinical Trial. JAMA Neurol. 2019;77:300-308.

13. Plosker GL. Stiripentol: in severe myoclonic epilepsy of infancy (Dravet syndrome). CNS Drugs. 2012;26:993-1001.

14. Lagae L, Sullivan J, Knupp K, et al. Fenfluramine hydrochloride for the treatment of seizures in Dravet syndrome: a randomised, double-blind, placebo-controlled trial. Lancet. 2019;394:2243-2254.

15. Pierce JG, Mithal DS. Fenfluramine: New Treatment for Seizures in Dravet Syndrome. Pediatr Neurol Briefs. 2020;34:8.

16. Skluzacek JV, Watts KP, Parsy O, et al. DS and parent associations: the IDEA League experience with comorbid conditions, mortality, management, adaptation, and grief. Epilepsia. 2011;52:95.

17. Sur L, Samasca G, Sur G, et al. Guillain-Barré Syndrome With a Peculiar Course: A Case Report. Cureus. 2021;13(5):e14905. 